\title{
Histoire des débuts de la géologie appliquée aux barrages en France
}

\section{J.L. BORDES}

20, rue de Madrid 75008 Paris

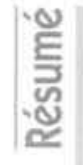

La géologie au sens où nous l'entendons est née à l'aube de l'ère industrielle, à la même époque où commençait le développement de la construction des grands barrages pour la navigation. l'alimentation des villes et la production d'énergie. Les constructeurs de barrages formés à une rationalité technique de plus en plus mathématisée, durent apprendre des géologues la complexité propre à toute science de la Nature, pour mieux appréhender les fondations de leurs structures. Cet apprentissage s'est fait non sans difficultés sous la nécessité de construire des ouvrages de plus en plus importants dans des sites souvent difficiles imposés par la topographie, Thydrologie et d'autres contraintes propres à la demande économique.

\section{History of the beginning of applied geology todays in France}

Geology as we now understand it, appeared at the same period as started the development of larges dam for waterways, water supply and energy. It was at the beginning of the industrial Revolution. Dam builders were educated in a rational way. strongly influenced by mathematics. To appraise the foundation problem, they were taugth by geologists the complexity of natural science. This practice became progressively customary painstakingly. Dam construction in larger and larger and difficult sites, was governed by economical demands and topographical and hydrological constraints. 
Le présent article a pour objet de retracer les débuts de la géologie appliquée aux barrages depuis le XVIII' siècle jusqu'aux environs des années 1920, qui marquent le début en grand de l'équipement hydroélectrique du territoire français. C'est au cours de cette période que s'étaient forgés les outils techniques qui ont permis à la communauté technique française de répondre avec succès aux défis qui lui étaient posés. Parmi ceux-ci, il y avait eu la mise en pratique de la géologie au service de la construction des grands barrages.

\section{1}

\section{Les origines de la géologie et son développement (Gouhau, 1987)}

\section{1}

\section{Les grandes dates}

C'est dans l'exploitation des mines par le biais de la minéralogie et de la pétrographie, que la géologie a fait son apparition dès le XV siècle. Elle ne trouvera son nom et son statut de science à part entière qu'á la fin du XVIII siècle, à partir du moment où fut brisé le tabou de l'age de la Terre. L'élaboration des uthéories de la Terren et donc de son histoire avait commencé en effet au XVII siècle, mais celles-ci se précisèrent au siècle suivant avec les cuvres de Gautier (1721), Bourguet (1729), et Buffon (1749 et 1778).

La stratigraphie particulièrement utile à l'ingénieur, fut le résultat des travaux de Nicolas Stenon (1669) quelque peu passés inaperçus lors de leur publication. S'ajoutèrent les apports de Werner (1744) pour la minéralogie, Lehmann (1756) sur la formation de la Terre, H.B. Saussure (1779) sur la description des Alpes, Cuvier (1812) pour la paléontologie, et W. Smith (1816) pour la classification des couches. On doit mentionner enfin C. Lyell (1830), dont le rōle fut considérable dans l'élaboration d'hypothèses de formation de la Terre, après celui de Hutton (1788 et 1795).

La géologie moderne naquit très rapidement entre 1810 et 1830 . Tout au long du XIX" siecle, le recueil d'observations et interprétations des scientifiques de toute l'Europe avec chacun leur génie propre, comme les Anglais les Allemands ou les Italiens, contribuerent au développement de cette science. Le traité de géognosie de J.F. d'Aubuisson, publié en 1819, présenta pour un public averti plus large l'exposé des connaissances de l'époque sur la constitution physique et minérale du globe terrestre. En 1849, d'Orbigny publia une classification des terrains en 28 étages. L'accumulation des observations avait obligé à revoir la classification sommairement définie à la fin du XVII' siècle.

En 1830 fut fondée la société géologique de France, celle d'Angleterre l'ayant été en 1827, après qu'un premier groupe de géologues se fut réuni à Londres dès 1807. Le premier congrès international de géologie se réunit à Paris en 1878 .

\section{2}

\section{Les premières cartes géologiques}

Fontenelle dès 1710 avait eu l'idée des cartes géologiques à partir des premiers travaux sur les fossiles. Un atlas minéralogique de la France amorcé par Guettard et Lavoisier fut interrompu par la Révolution. Cuvier et Brongniart publièrent une carte "géognosique " des environs de Paris au 1/200000 en 1811, et datèrent les couches d'après les fossiles qu'ils y trouvèrent. Le terme de carte géologique apparait en 1816, lorsque W. Smith publie une carte en 15 feuilles de l'Angleterre, cu pays de Galles et partiellement et de l'Écosse à I'échelle d'environ 1/500 000. La carte géologique de la France au 1/500 000, sous la direction d'Élie de Beaumont ne fut complétée seulement qu'en 1841.

\section{3}

\section{L'enseignement en France}

La géologie fut enseignée à partir de 1793, gràce à la création d'une chaire au Musèum d'histoire naturelle, dont le premier titulaire fut Faujas de Saint-Fond. Elle constitua la matière d'un cours en 1794 à l'École des mines de Paris fondẻe en 1783, en 1823 à l'École des ponts et chaussées et en 1830 à l'École centrale dès la fondation de cette dernière. Mais cet enseignement a souvent souffert d'un académisme certain.

L'École de géologie de Nancy fut créée en 1908 et répondait, à l'initiatives des milieux universitaires et industriels de la région, au besoin d'avoir des géologues formés en vue de l'application de la géologie à différents domaines industriels et en particulier minier. C'était aussi la période de développement des aménagements hydroèlectriques. Il fallait faire le pont entre la demande des ingénieurs de génie civil et les réponses que pouyait apporter la géologie et faire bénéficier les deux parties des apports de chacune des spécialités, l'aspect quantitatif d'un cōté, historique et spatial de l'autre.

On devine à la lecture de certains textes du XIX" siècle qu'il y avait une répugnance de la part de certains geologues à sortir du domaine purement scientifique de la géologie. Mais comme l'a écrit Jean Goguel: aIl n'y a pas - quant à la démarche intellectuelle qui s'efforce de dépasser les observations, ce qui est le propre d'une science - une géologie de l'ingénieur qui soit distincte la géologie, au sens général. Il y a seulement des problèmes plus ou moins locaux, où l'on s'efforce de pousser l'analyse dans un plus ou moins grand détail. ’

\section{2}

\section{Panorama de l'évolution des barrages du Moyen Age jusqu'au début du $x x^{e}$ siècle en France}

\section{2,1}

\section{Le Moyen Age}

En France, la pratique de la construction des barrages trouve son origine dans celle des digues des étangs monastiques réalisés dès le haut Moyen Age à des fins piscicoles. Ils furent rapidement utilisés simultanément à d'autres finalités, en particulier énergétique, pour faire fonctionner des moulins à blé, puis des 
soufflets de forge et d'autres mécanismes industriels, participant à l'équipement en moulins du territoire français qui atteignit son apogée à la fin du Xill siècle, et se maintint jusqu'au début du XIX ${ }^{e}$ siècle. La digue de Jugon dans les Côtes-d'Armor est un très bel exemple vieux de huit siècles. C'était dès sa construction un ouvrage à but multiple qui était utilisé pour la pisciculture, assura le fonctionnement de deux moulins jusqu'au début du XX" siècle et d'une forge un peu plus à l'aval. De plus, le plan d'eau ainsi créé, isolait pour partie le château du seigneur local.

\section{2.}

\section{La proto-industrie}

Le développement à partir du Xv' sjècle du procédé indirect de production du fer, la contrainte de la continuité de la prociuction, I'influence des outils de transformation de l'énergie, favorisèrent la réalisation de réservoirs de dimensions non négligeables appelés étangs de forge, qui se multiplièrent aux Xvile et XVIII siècles. Hs étaient souvent associés en chapelet afin d'augmenter leur capacité régularisatrice. On dispose d'un très riche ensemble de réalisations en France (Berry et Centre, Bretagne et le pays de Châteaubriand, Châtillonnais, Franche-Comté, Normandie, Périgord, Rhône-Alpes), qui ont été inventoriées dans le cadre de I'Inventaire général des monuments et richesses artistiques de la France. II ne faut pas oublier le cas particulier des barrages pour les mines, par exemple la digue de la Noie à Poullaouèn, qui sont en même temps le témoignage de transfert technologique (du Harz, de la Suède et d'ailleurs) et de l'intégration de techniciens étrangers. Les retenues qui existent encore, constituent une très grande partie des plans d'eau français, qui doit-on le rappeler sont à plus de $90 \%$ le résultat du travail de l'homme.

\section{3}

\section{De la construction des canaux de l'Ancien Régime à la première industrialisation}

La politique de construction des canaux, commencée sous Francois Jer trouvait sa raison dans la nécessité de résoudre les difficultés du transport sous l'Ancien Régime, en particulier pour assurer le ravitaillement en blé et en bois de chauffage des grandes villes de l'époque. Le transport par eau présentait un avantage énergétique tout à fait considèrable, par rapport aux autres moyens. Cette politique se traduisit par le primat de la navigation sur les rivières navigables et flottables, bien que leur utilisation énergétique füt loin d'être négligeable. L'alimentation, des canaux à point de partage exigea des réservoirs de grande capacité, à partir de la construction du canal de Briare (1642). Le réservoir de Saint-Ferréol (1675) pour l'alimentation du canal du Midi avait une digue en terre qui fut la plus haute du monde pendant quelque trois cents ans. La progression de la construction des canaux fut néanmoins lente, La première industrialisation accéléra considérablement son rythme. Le programme de construction s'acheminait vers sa fin, lorsque commença le développement du chemin de fer, dont la concurrence mal maitrisée fut très dommageable aux voies navigables.
Dans l'ensemble pour cette période, la plupart des barrages étaient la plupart du temps en terre et de hauteur modeste tout au plus de $10 \mathrm{~m}$. Toutefois, en 1815 on dénombrait en Europe et dans l'Amérique sous influence hispanique, 20 ouvrages de plus de 20 mètres de hauteur, toujours en service de nos jours, construit pour le plus ancien au XII! siècle. Leurs finalités, multiples pour certains, concernaient dans l'ordre d'importance, l'énergie, l'irrigation et enfin l'alimentation de canaux pour la navigation (Schnitter, 1994).

\section{4}

\section{L'affirmation des finalités énergétiques dans la première industrialisation}

C'est à Aix-en-Provence que fut construit le premier barrage-voûte de l'ère industrielle, le barrage Zola (1854). Destiné en premier lieu à l'alimentation en eau de la ville, il avait aussi une fonction énergétique. Malgré la réussite technique qu'il représentait, il passa presque inaperçu, et n'eut aucune postérité immédiate. Dans la région de Saint-Étienne, l'approvisionnement en eau des villes et le soutien des étiages pour les usines furent le moteur d'un saut technologique constitué par la construction du barrage en maconnerie du Furens (1866), qui fut reproduit dans les vallées voisines, Le barrage du Furens, le plus haut barrage construit alors par I'homme, devint un modele pour le monde entier. Ces entreprises furent caractérisées par la maitrise d'ouvrage d'investisseurs privés ou de collectivités locales. Dans les Vosges alsaciennes, l'aménagement de lacs par surélévation, à la seule finalité énergétique, fut également le résultat de l'action des associations syndicales et des acteurs privés. Enfin, les lacs pyrénéens furent aménagés par les ingénieurs du gènie rural à la fois à des fins d'irrigation et ènergétiques.

\section{5}

\section{Les premiers barrages hydroélectriques dans la deuxième industrialisation}

C'est dans le cadre de l'utilisation des hautes chutes des Alpes et dans les Pyrénées que furent réalisés les premiers aménagements hydroélectriques à la fin du XIX" siècle. Dans les Alpes, la demande énergétique des usines électrochimiques et électrométallurgiques fut déteminante. Les usines d'éclusée du piedmont Nord du Massif central. avec une finalité commerciale, dont Rochebut (1909) est un modèle, firent du Massif central un terrain d'expérimentation (Les Fades, Miodex, la Bourboule) qui devait trouver son aboutissement après 1920, Cette mẻme période avait été celle de la construction des premières usines de plaine, Jonage (1899), et sur une grande rivière d'un barrage quasi au fil de l'eau, Tuilière (1908), conçues toutes les deux pour l'alimentation de grosses agglomérations.

\section{6}

\section{La crise énergétique de la Première Guerre mondiale et la loi de 1919}

L'occupation des zones charbonnières du Nord de la France par les troupes allemandes pendant la Pre- 
TABLEAU1 Quelques barrages remarquables dans I'histoire en France.

Some noteworthy historic dams in France.

\begin{tabular}{|c|c|c|c|c|c|c|c|c|}
\hline $\begin{array}{c}\text { Nom } \\
\text { du barrage }\end{array}$ & $\begin{array}{c}\text { Arnée } \\
\text { de mise } \\
\text { en service }\end{array}$ & Cours d'eau & $\begin{array}{l}\text { Ville } \\
\text { la plus } \\
\text { proche }\end{array}$ & Département: & Typel & $\begin{array}{l}\mathrm{H}^{\mathrm{2}} \\
(\mathrm{m})\end{array}$ & $\begin{array}{c}\mathrm{Vr} \\
\left(10^{6} \mathrm{~m}^{3}\right)\end{array}$ & Concepteur \\
\hline Jugon ${ }^{4}$ & 1240 & La Rosaie & Jugon & Côtes-d'Armor & Terre & 8,5 & 2.5 & \\
\hline Saint-Ferréol ${ }^{5}$ & 1675 & Le Laudot: & Revel & Hte-Garonne & Terre & 36 & 6,7 & $\begin{array}{c}\text { Clerville } \\
\text { Andréossy }\end{array}$ \\
\hline La Noiefi & 1752 & & Huelgoat & Finistère & Terre $^{7}$ & 15 & 1.5 & Koenig \\
\hline Zola ${ }^{0}$ & 1854 & L'Infernet & Aix-en-Provence & Bouches-du-Rhōne & Voùte & 42 & 2,5 & F. Zola \\
\hline Le Furens" & 1866 & Le Furens & Saint-Étienne & Loire & Poids & 56 & 1,35 & Graeff, Delocre \\
\hline La Bourboule ${ }^{16}$ & 1896 & La Dordogne & La Bourboule & Puy-de-Dôme & Poids & 23 & 0.5 & Tainturier \\
\hline Jonage 11 & 1899 & Le Rhōne & Lyon & Rhóne & V & 21 & & Raclet \\
\hline Sauviat & 1903 & Le Miodex & Thiers & Puy-de-Dóme & Poids & 27 & 0.7 & Fay \\
\hline Tuilière & 1908 & La Dordogne & Bergerac & La Dorciogne & V & 33 & 5 & GTM, SGE et Palaz \\
\hline Rochebut & 1909 & Le Cher & Montluçon & Allier & Poids & 50 & 26 & Mialaud \\
\hline Les Facles: & 1917 & La Sioule & Queuille & Puy de Dóme & Poids & 34 & 6 & \\
\hline
\end{tabular}

7 Poids barrage en maçonnerie, Vi barrage en rivière à vanne

${ }^{2} \mathrm{H}$ : hauteur du barrage au-dessus des fondations.

Vr: volume de la retenue.

- Cet ouvrage n'est cité qu'à titre d'exemple parmi d'autres cas.

Record du monde de hauteur pour les barrages en remblaf

- Ouvrage désaffecté en 1860, toujours existant.

7 La digue de la Noie était un ouvrage original, construit suivant les pratiques du Harzet comprenant une partie amont en enrochements rangés, un noyau central vertical étanche. une recharge aval en terre.

"Premier barrage voùte au monde de l'ère industrielle.

"Record du monde de hauteur de barrage de tout type.

MLa ville de la Bourboule fut une des premières en France à être éclairèe à lélectricité

11 L'usine-barrage de Cusset fut l'usine hydroélectrique la plus importante du monde (22 000 CV) aprés celle cies chutes du Niagara (50 000 CV).

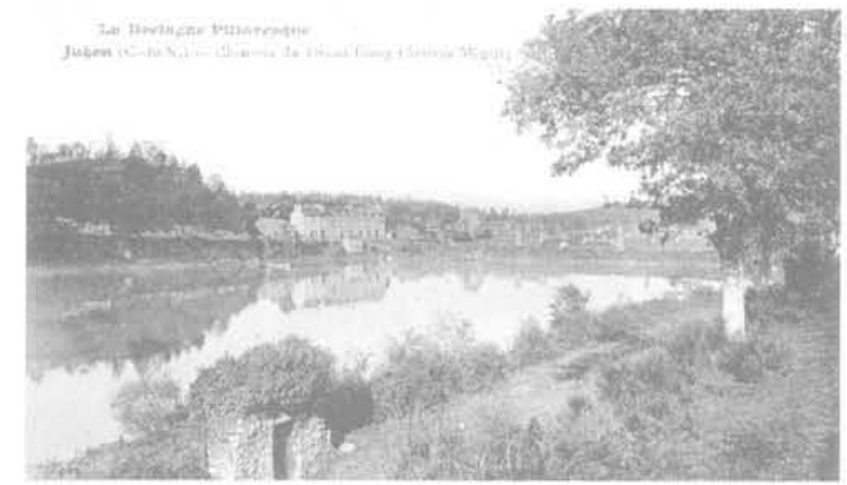

vers 1240

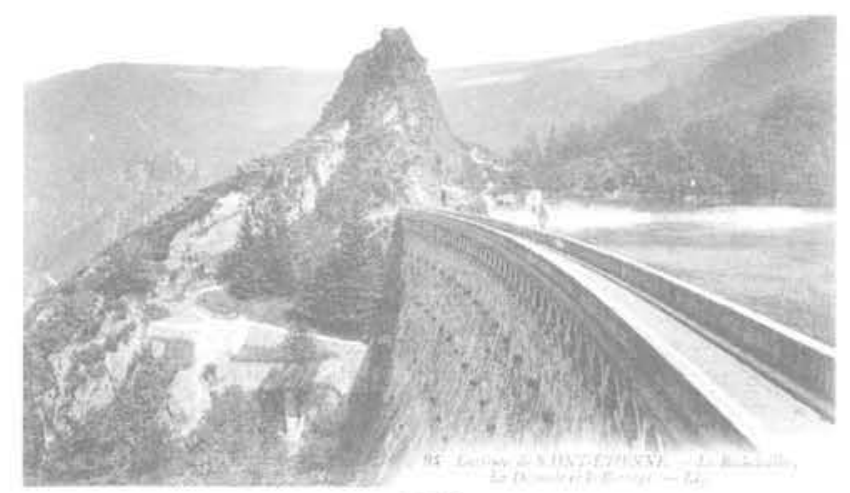

1866

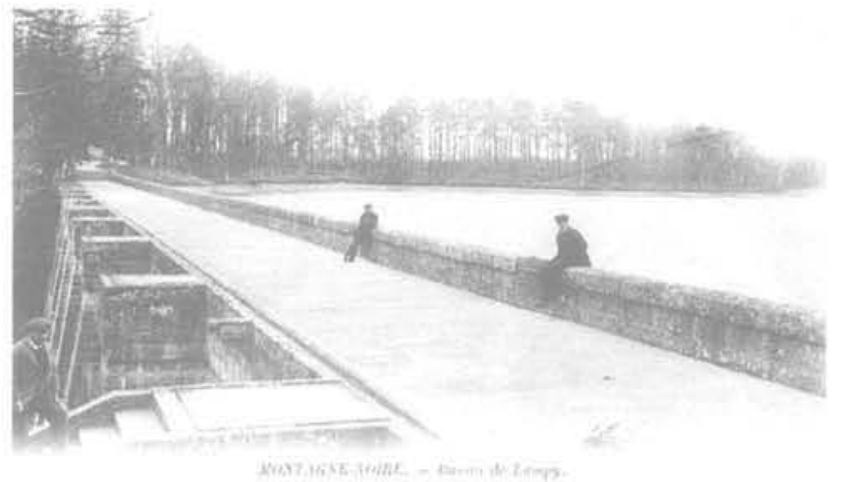

1782

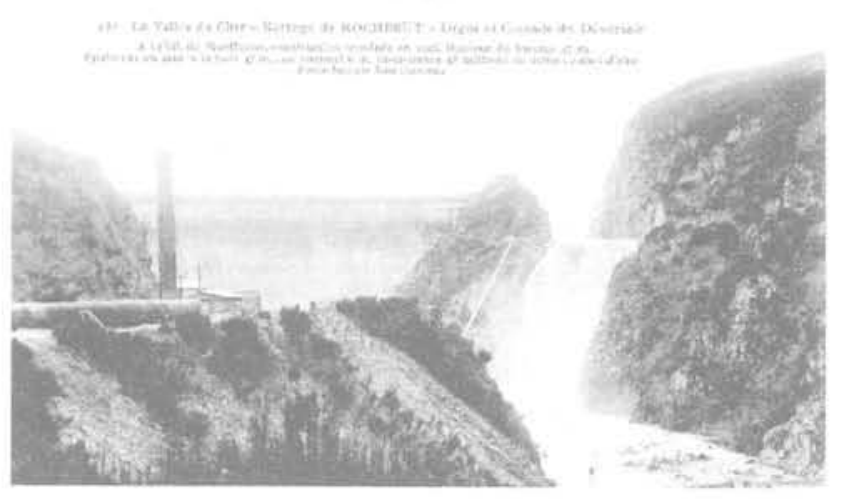

1909

FIG.1 Cartes postales de quatre barrages remarquables du $\mathrm{XII}^{\circ}$ au début du XXe siècle. Postcards of four noteworthy dams from Xuth to Xxth centuries. 
mière Guerre mondiale, provoqua une crise énergétique de grande ampleur. La prise de conscience de la valeur des ressources hydroélectriques du pays, déjà sensible depuis 1900, n'en devint que plus forte. La prospection des sites fut accélérée. La construction d'aménagements fut facilitée. Mais cette crise conduisit à l'élaboration d'une véritable politique d'indépendance énergétique. Elle aboutit pour ce qui était du volet hydraulique à une véritable nationalisation de l'énergie des rivières dont les modalités d'utilisation étaient définies dans la loi du 16 octobre 1919. Elle fut à l'origine de la construction des barrages à finalité énergétique dès l'après-guerre mais surtout après 1945. Si les problèmes ne changèrent pas de nature au plan qualitatif, le nombre des ouvrages construits en quelques dizaines d'années, le volume moyen des retenues, la superficie des bassins versants drainés, la puissance installée des aménagements, furent augmentés dans des proportions telles que la réalisation d'équipement hydroélectrique jusqu'alors limité, devint une activité tout à fait considérable au plan natienal. La date de 1920 marque le début d'une autre ère.

\section{3}

\section{Géologie et barrages}

\section{1}

\section{Les premières attentes des ingénieurs}

Il n'y a guère de considérations géologiques dans les premiers documents écrits sur l'art des barrages, comme le traité de l'abbé Bossut (1764), ou l'ouvrage de Lalande (1778). Toutefois le traité d'exploitation des mines de Délius traduit de l'allemand (1778), contient un chapitre très intéressant sur la construction des barrages destinés à faire fonctionner les systèmes d'exhaure des mines. Dans cette synthèse des pratiques des zones minières du Harz et de la Bohème, on note un souci très méticuleux de la reconnaissance des fondations, de leur structure pourrait-on dire, qu'on ne retrouve pas dans les traités ultérieurs relatifs à la navigation intérieure de C.J. Minard (1842), L.C. Mary (1851), professeurs à lÉcole des ponts et chaussées. Minard allait jusqu'à dire qu'il ne fallait pas s'abuser sur le degré d'utilité de la géologie pour aider l'ingénieur à prévoir les terrains qu'il allait rencontrer dans les grandes tranchées et souterrains.

Malgré cette discipline encore balbutiante à leur disposition, les ingénieurs ou plus simplement les constructeurs de digues des étangs de forge au XVII" siècle attendaient de la «géognosie» qu'elle les aidât à trouver des cuvettes de retenues étanches, et des carrières de matériaux. Dans la pratique des constructeurs de barrage, la préoccupation d'une bonne approche de l'environnement géologique devait être la règle depuis longtemps, sans être toutefois une priorité. Le relevé de détail des fouilles du barrage du Lampy effectué en 1779 (Archives du canal du Midi), témoigne d'une approche rationnelle des problèmes techniques à résoudre, avant les avancées fondamentales de la résistance des matériaux dans les années 1820 à 1830. Toutefois, l'appréciation de la nature des couches n'est pas exempte d'erreurs, et celle de leur résistance relève de la pénétration plus ou moins grande du pic à rocher dans les terrains rencontrés.

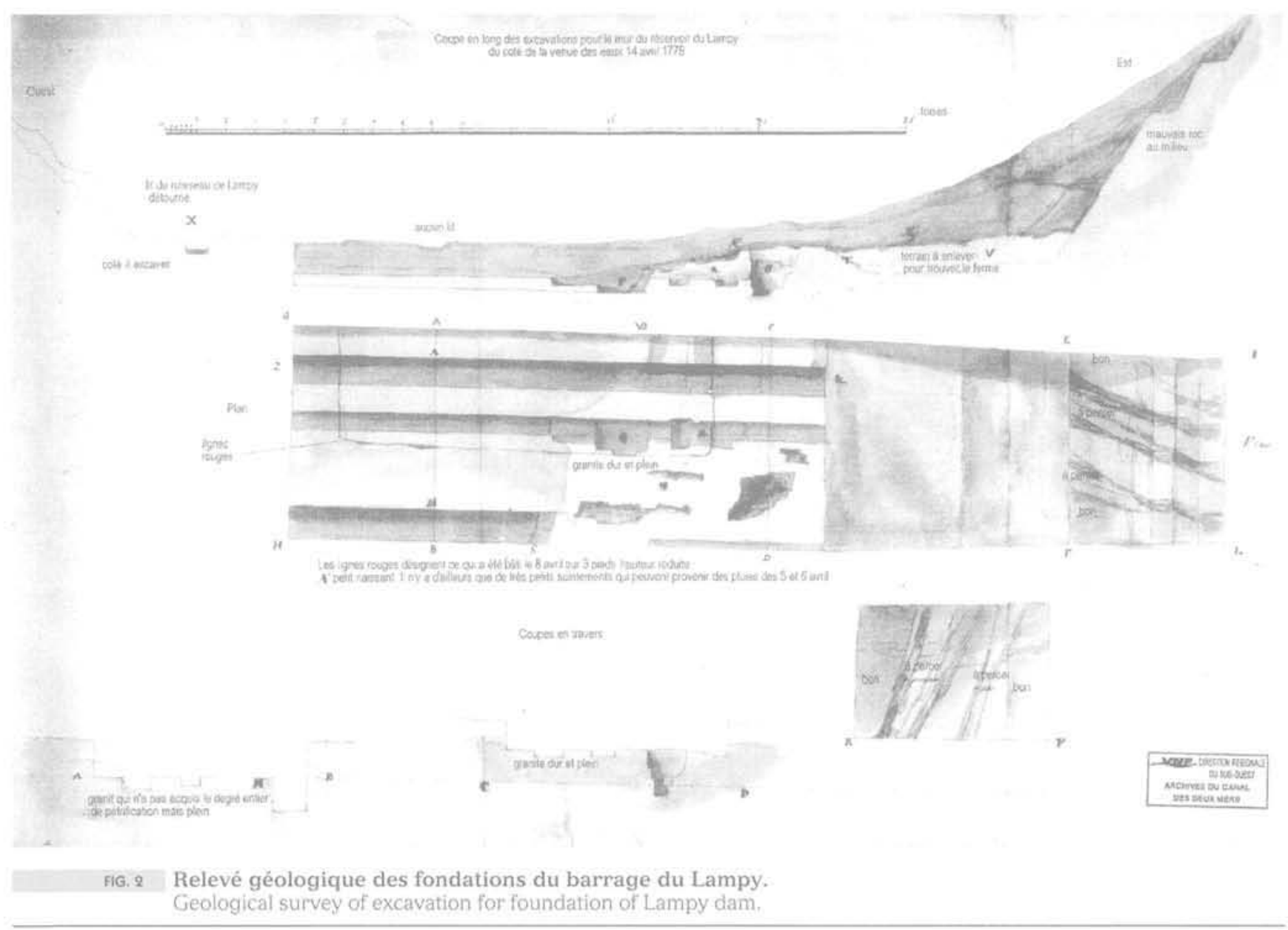


La construction du tunnel de Pouilly (1824-1832) au point de partage du canal de Bourgogne fut l'occasion d'investigations très poussées avec un puits de $27,40 \mathrm{~m}$ de profondeur, exécuté en 1810 , repris en 1818 et descendu jusqu'à $54,60 \mathrm{~m}$ de profondeur. Un puits d'épreuve de $12 \mathrm{~m}$ de profondeur, avait été creusé en 1752. Le tunnel de 3330 mètres de lonqueur, de 6,2 mètres d'ouverture sous 50 mètres de couverture. fut exécuté au moyen de 32 puits verticaux d'attaque, groupés deux par deux à $40 \mathrm{~m}$ de distance, chaque groupe étant espacé de $200 \mathrm{~m}$. La connaissance détaillée des terrains s'étendait sur une épaisseur de $150 \mathrm{~m}$, divisée en 8 couches. Les archives du canal aux Archives nationales contiennent des documents iconographiques tout à fait remarquables sur la zone, dont une carte géologique datant de 1835 à l'échelle du 1/25000, signé Lacordaire.

Le tunnel de Pouilly n'était qu'un ouvrage parmi ceux nécessaires pour compléter le canal. Cing barrages d'alimentation du bief du point cle partage furent conçus en même temps en 1829, dont le barrage de Grosbois qui a posé de très gros problèmes de stabilité dès sa mise en exploitation. Ces ouvrages firent l'objet de deux ensembles d'avant-projets au sens que nous donnons à ce terme à notre époque. Dans celui relatif à Grosbois, plus de six pages sur cent sont consacrées à la description de la géologie de la zone et de ses conséquences sur la perméabilité des cuvettes, la circulation des eaux souterraines, la réalisation des rigoles, et bien évidemment sur la nature de la fondation du barrage de Grosbois. Tant pour le calage du niveau du tunnel que pour l'implantation des retenues et des rigoles, la géologie a joué un rôle très important.

Nous avons également trouvé aux Archives nationales, des documents géologiques très complets établis de 1846 à 1848 pour l'étude d'un réservoir à créer à Saint-Bonnet sur le tracé du canal de la Marne au Rhin. Le réservoir fut abandonné à cause des craintes étavées par l'étude géologiques concernant l'étanchéité de la cuvette.

\section{Le problème des moyens de reconnaissance}

Les difficultés qui surcirent lors des travaux vinrent de l'expérience très insuffisante acquise à l'époque, et de l'absence d'outils de connaissance des propriétés mécaniques des matériaux, l'ignorance du rôle de la pression de l'eau dans les sols, qui ont conduit les ingénieurs à se méprendre sur la résistance et le comportement à attendre du terrain de fondation (Bordes, 1999).

La pratique de la géologie dans les barrages s'est heurtée pendant longtemps à la faiblesse des moyens de reconnaissance. Durant une lonque période, ceux-ci furent limités à des puits et galeries chaque fois que cela était possible et que l'importance de l'ouvrage le justifiait. ce qui fut exceptionnel jusqu'à la fin du XIX'siècle.

Les sondages bénéficièrent d'abord des progrès faits dans les recherches de nappes artésiennes à la fïn du $\mathrm{XVII}^{e}$ siècle et au début du XIX ${ }^{\circ}$ siècle. La profondeur de $500 \mathrm{~m}$ fut atteinte peu après 1840 au forage artésien de Grenelle. Mais ce n'est qu'au début đu XX siècle et plus particulièrement après 1920 , gràce à la prospection minière et pétrolière, que la géologie appliquée au génie civil put disposer de moyens efficaces de reconnaissance, et tout particulièrement d'outils de prélèvement.
Bien que la pratique des reconnaissances fut recommandée avec insistance depuis des temps aussi lointains que celui de Perronet en 1769 et probablement avant, les maitres d'ouvrage arguant des coûts et des délais, ont toujours cherché à réduire ce type de dépense, au risque de ne pas avoir les informations requises.

C'est ainsi que la première coupe géologique du site de Bouzey de 1876 est déclarée inadécuate par les ingénieurs à la lumière des conditions du premier accident de 1884, accident dủ à la nature des fondations et à l'effet des sous-pressions dont le rôle à l'époque était très mal compris. Pendant les travaux de construction du mur parafouille, une coupe géologique avait été dressée en 1879. Elle put être précisée par un puits de 17 in de profondeur creusé au droit de la zone endommagée en 1884. La figure 3 donne la coupe du terrain sous le barrage, et indique la position et l'ouverture des fissures, après le déplacement vers l'aval d'une partie du barrage. La lecture des rapports des ingénieurs relatifs à cette affaire montre que la géologie n'était pas assez intégrée à leur pratique. On peut penser que les progrès dans les méthodes de calculs, l'amélioration constante des matériaux de construction ont détourné les ingénieurs de l'observation de l'environnement géologique, et du comportement des ouvrages. C'était une régression par rapport à la démarche des ingénieurs du canal de Bourgogne, cinquante ans plus tôt. Ils ont eu l'illusion vite dissipée que les problèmes de fondations pouvaient être résolus comme ceux du corps du barrage. L'ère des laboratoires, qui allaient permettre de garder ou de retourner à une approche expérimentale, avait tout juste commencé (Bordes, 2000). Les ingénieurs, français plus spécialement, très marqués par leur formation mathématique, avaient dés difficultés pour comprendre la complexité des données de la nature.

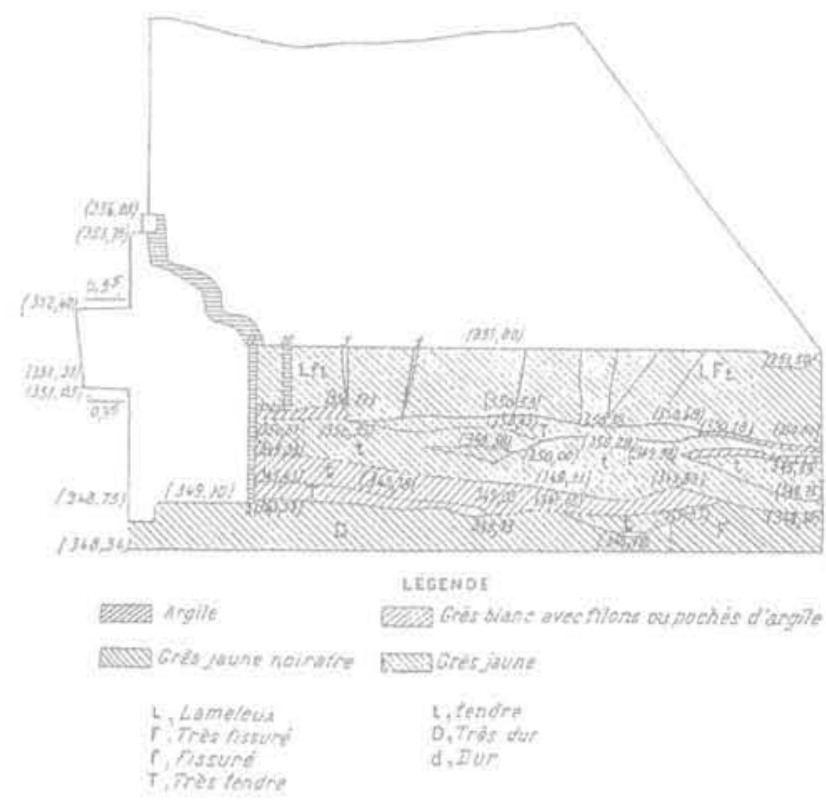

FIG.3 Coupe géologique de la fondation du barrage de Bouzey dans la zone rompue en 1884 (Le Génie civil, tome XXXI, n ${ }^{\circ} 12$. 1897, p. 187).

Geological section of the foundation of Bouzey dam in the vicinity of the rupture in 1884. 


\section{La géologie appliquée devient de plus en plus importante à la fin du XIXe et au début du XX $x^{e}$ siècle}

Nous avons décrit au début de l'article, l'évolution de la construction des barrages suivant une périodisation qui s'articulait sur les différents épisodes de l'industrialisation. L'histoire technique de la conception des barrages peut être divisée en une grande période allant du début du Xvur siècle jusqu'à la réalisation du Furens (1866), puis une phase s'étendant jusqu'à la deuxième rupture de Bouzey (1895), suivi par une période dont la limite peut ètre fixée à la parution de la circulaire ministérielle de 1923 sur les barrages de grande hauteur. Il ne nous semble pas possible de définir une périodisation similaire des étapes de l'évolution de la géologie appliquée aux barrages, à cause de la nature mème descriptive et synthétique de cette science. Il faut donc rechercher les apports des géologues, dans le cadre des demandes qui leur ont été faites, des épisodes particulièrement significatifs. Tout au long du XIXe siècle, le nombre de grands barrages (hauteur supérieure à $15 \mathrm{~m}$ au-dessus des fondations) construits en France est modeste : 36 au total, à peine plus d'un tous les trois ans. Beaucoup ne posèrent aucun problème particulier de fondations. Mais peu avant 1900, le développement de l'hydroélectricité et celui de l'alimentation en eau des villes, changèrent de façon très sensible les données du problème. Ils obligèrent les ingénieurs à prendre en considération des sites de plus en plus importants et, par là même, de plus en plus complexes au plan géologique. L'équipement hydroélectrique en montagne avec toutes les dérivations et conduites associées fut un facteur d'amplification des problèmes. Trois nous semblent particulièrement offrir des enseignements ayant valeur d'exemple.

- Le site de Serre-Ponçon fut inventé dès 1856 pour protéger la vallée contre les crues, année au cours de laquelle des premiers sondages peu profonds (moins de $10 \mathrm{~m}$ ) furent exécutés. Des études et reconnaissances furent entreprises à partir de 1897, cette fois-cj pour alimenter les zones irriguées de la Provence: Le site était caractérisé par l'existence d'un sillon alluvial très profond dont la profondeur était sans cesse estjmée à la hausse au fur et à mesure de l'avancement des sondages très difficiles à exécuter à cause de l'insuffisance technique des moyens de l'époque. Alors que l'on croyait avoir trouvé le rocher à $42 \mathrm{~m}$ de profondeur en 1899, le projet de barrage est repoussẻ à plus tard, bien que des études fussent continuées. En 1910 avec la création de la société pour la régularisation de la Durance pour produire de l'électricité, les études et les reconnaissances prenaient un nouveau départ. Un puits de $63,10 \mathrm{~m}$ fut creusé et des sondages à partir d'un rameau de $75 \mathrm{~m}$ de long sont exécutés en 1913. Malheureusement un accident provoqua l'envahissement par les eaux et stoppa les reconnaissances (Fig. 4). De nouveaux sondages en discussion dès 1913 immédiatement après l'accident, ne purent ètre exécutés qu'en 1925. Ils conduisirent à l'abandon provisoire du site en 1927. Des études furent timidement conduites en 1941. En 1947 reconnaissances et études reprirent jusqu'à la réalisation de l'ouvrage, dont certaines suivant le schéma prévu trente ans plus tôt (sondages rayonnants à partir du puits de reconnaissance). Le nom d'Ivan Wilhelm doit rester associè à cette ceuvre. Il en fut le premier projeteur, défendit le projet tout au long de sa vie, et eut la chance au soir de celleci devoir le début des travaux.

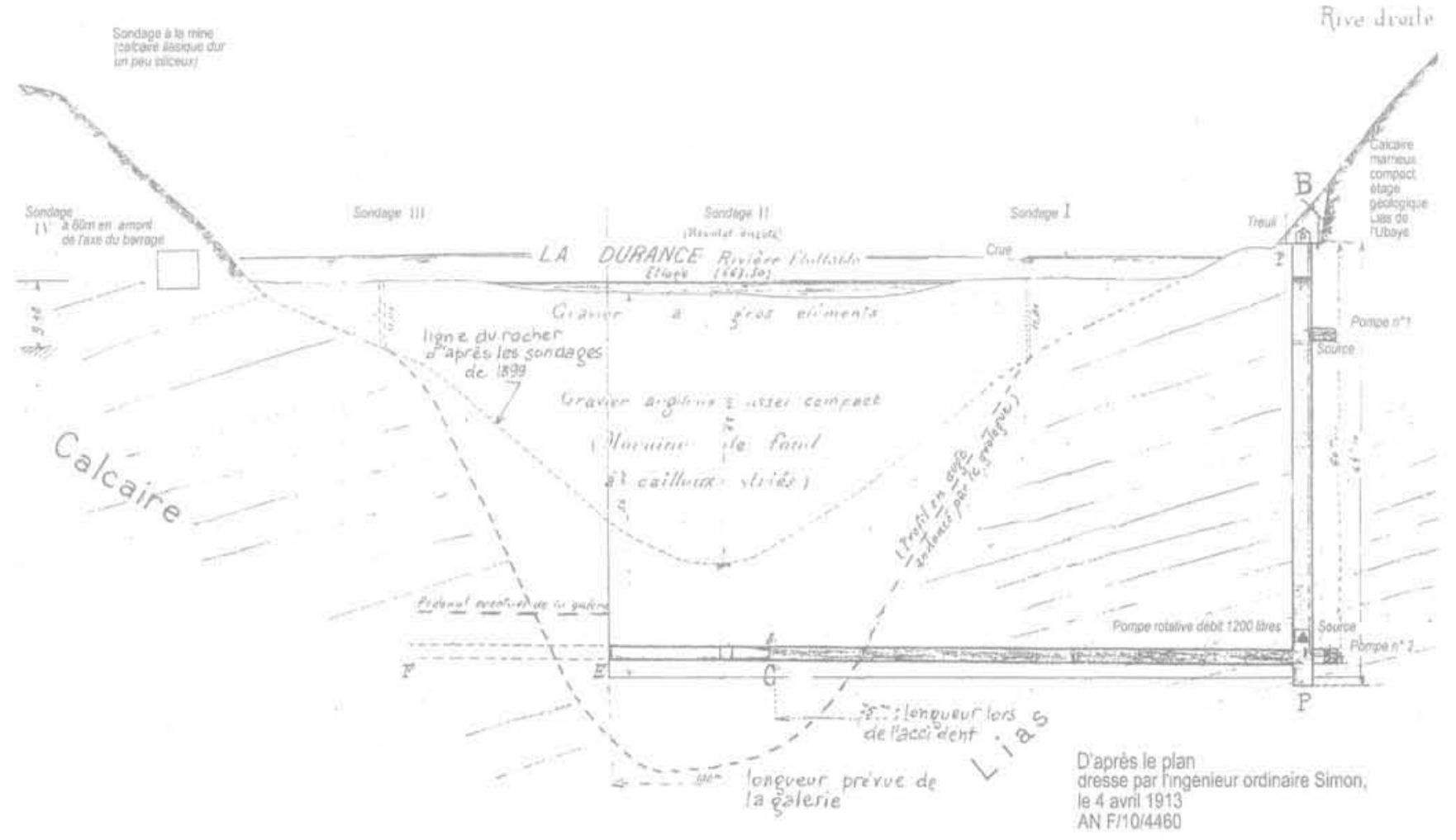

FG. 4 Reconnaissance du sillon alluvial au site de Serre-Ponçon en 1913. Investigation of sub-alluvial thalweg at Serre-Ponçon dam site in 1913. 
Dès la fin du XIX siècle, Zurcher assisté d'autres géologues travailla en vue de déterminer la structure du site et en particulier la géométrie du sillon alluvial, et le régime des nappes. Kilian examina le site en 1917, et participa à l'interprétation des données recueillies au cours des reconnaissances, ainsi qu'à la définition des reconnaissances ultérieures.

- Le barrage de Génissiat fut proposé pour la première fois en 1902, par Blondel Harlé et Mahl, si on ne tient pas compte des propositions un peu utopiques de la fin du XVIII et du début du XIXe siècle. Le but était d'alimenter la région parisienne en électricité, Sa faisabilité fut mise en question à cause de l'épaisseur du remplissage alluvial du sillon et de sa profondeur estimée en relation avec la tectonique du site, ainsi que le risque des circulations potentielles d'eau dans le massif calcaire dans lequel était inscrite la retenue. Martel le spéléologue bien connu, Lugeon le grand géologue suisse, et Kilian, effectuèrent plusieurs missions, et rédigèrent de nombreux rapports à partir de 1910, dans le cadre d'une commission géologique constituée d'abord pour la connaissance du site de Bellegarde puis de celui de Génissiat. Plusieurs techniques de reconnaissances furent mises en œuvre au cours des années qui furent nécessaires pour faire les études.

- Le barrage de Dardennes a été conçu pour l'alimentation en eau de la ville de Toulon. Il fut mis en eau en 1914. Les problèmes d'étanchéité exigèrent la réalisation d'une coupure étanche en prolongement du barrage au moyen d'un masque de 2,1 m d'épaisseur et de $170 \mathrm{~m}$ de longueur, complété par des injections dans des forages exécutés à la barre à mine (Fig. 5). Les géologues Bertrand, Zurcher, Vasseur et Kilian furent appelés en consultation.

Pour tous ces ouvrages, nous n'avons fait qu'effleurer les problèmes. Notre propos n'est pas d'en faire l'historique, mais de montrer que ceux-ci constituèrent des étapes qui ont marqué l'évolution de la géologie appliquée aux barrages. Sur deux de ces sites les plus importants, études et travaux s'étendirent sur plusieurs dizaines d'années. Ces sites constituèrent de véritables laboratoires au sens que nous donnons à ce mot, oủ s'élaborèrent des méthodes d'observations et de reconnaissances. Ingénieurs et géologues apprirent à mieux collaborer. Les premiers apprirent des seconds à penser à trois dimensions et à intégrer le temps dans leurs analyses, les seconds à comprendre le souci de modélisation et de dimensionnement des premiers. Le géologue spécialiste des barrages est né au début du siècle passé. M Lugeon (1870-1953) estimait que sa génération en avait été témoin.

\section{4}

\section{Les injections (Glossop, 1960-1961)}

La technique des injections est associée à la géologie des barrages. Celles-ci furent dès 1805 un des domaines privilégiés de l'innovation des techniciens français dans le génie civil. Mais pour les barrages en maçonnerie fondés sur le rocher, elles se développèrent seulement à partir de 1893 aux États-Unis après un premier essai en 1876 en Angleterre. Elles ne devinrent une pratique courante que peu avant la Première Guerre mondiale. M Lugeon définit les premières règles pour conduire les injections dans le rocher.

L'injection des terrains meubles s'est développée à partir des techniques de fonçage des puits de mines dans la traversée des terrains superficiels au début du $\mathrm{XX}^{\mathrm{E}}$ siècle. Le tube à manchette inventé par Ischy date de 1927. Il fut mis en œuvre pour l'injection des sables de Bou Hanifia en Algérie dès 1934, dans les fondations du batardeau de Génissiat en 1936, dans celles du batardeau du Sautet en 1937, avant d'ètre utilisé avec le succès que l'on sait à Serre-Ponçon dans les années 50 .

\section{4}

\section{En conclusion, l'évolution après 1920}

Les maîtres d'ouvrage et maitres d'ceuvre, administrations, sociétés d'électricité, bureaux d'études, avaient fait appel aux géologues universitaires à la fin du XIXe siècle. L'école grenobloise était particulièrement brillante. Nous avons déjà cité le nom de W. Killian qui intervint sur les sites de Serre-Ponçon, Génissiat et Dardennes, auxquels succédèrent P. Lory, M. Gignoux et Reynold Barbier. L'administration eut ses spécialistes comme Zurcher au début du siècle passé. Les sociétés d'électricité eurent souvent leur propre géologue. EDF créa un service géologique en 1946 dont le premier responsable fut.J. Crosnier-Leconte. Des spécialistes étrangers intervinrent lorsque ce fut nécessaire. Le nom de Maurice Lugeon qui fut conseiller à Génissiat et sur de nombreux autres sites en France n'est pas le moindre.

Malgré les exemples que nous avons cités, et l'ancienneté de certains, la géologie n'était pas le premier souci des ingénieurs constructeurs de barrages. Ce fut un long apprentissage à cause de la nature même des problèmes géologiques qui empêchent les généralisations abusives et donc le dogmatisme. Seule l'expérience enseigne. Il faut donc du temps. Ce ne pouvait ètre qu'à partir d'un grand développement des constructions hydroélectriques dans le cas de la France que pouvait se constituer un savoir-faire. Plusieurs auteurs le soulignent. L. Bertrand dans son article de 1930 à l'occasion du centenaire de la société géologique de France, détaillait quels pouvaient être les apports de la géologie. Les livres de M. Lugeon (1933) et de M. Gignoux et R. Barbier (1955), en dehors de l'expérience technique qu'ils présentent et des enseignements qu'ils en proposent, sont de véritables livres de I'histoire technique de la géologie. Car comme les auteurs l'expliquent très bien, l'accumulation d'observations est indispensable pour constituer un outil technique qui puisse aider l'ingénieur. Le côté naturaliste du géologue est un apport essentiel pour l'ingénieur. Cette expérience fut dans le cas particulier de la France, enrichie de celle acquise outre-mer par les ingénieurs français. Une mention spéciale doit être faite de l'expérience algérienne qui dans les années 20-40 fut exceptionnellement féconde dans le domaine de la géologie et de la mécanique des sols. Les conditions très difficiles des sites obligèrent les ingénieurs à se surpasser, ce qui bénéficia par la suite à la métropole.

La résolution du problème de la seule étanchéité auquel fut souvent limitée la question première des ingénieurs, fut dépassée. Au fur et à mesure de l'augmentation de la taille des ouvrages, la connaissance de la structure géologique des sites et des conditions de sa formation, a aidé les ingénieurs à comprendre le fonctionnement mécanique de la fondation des bar- 


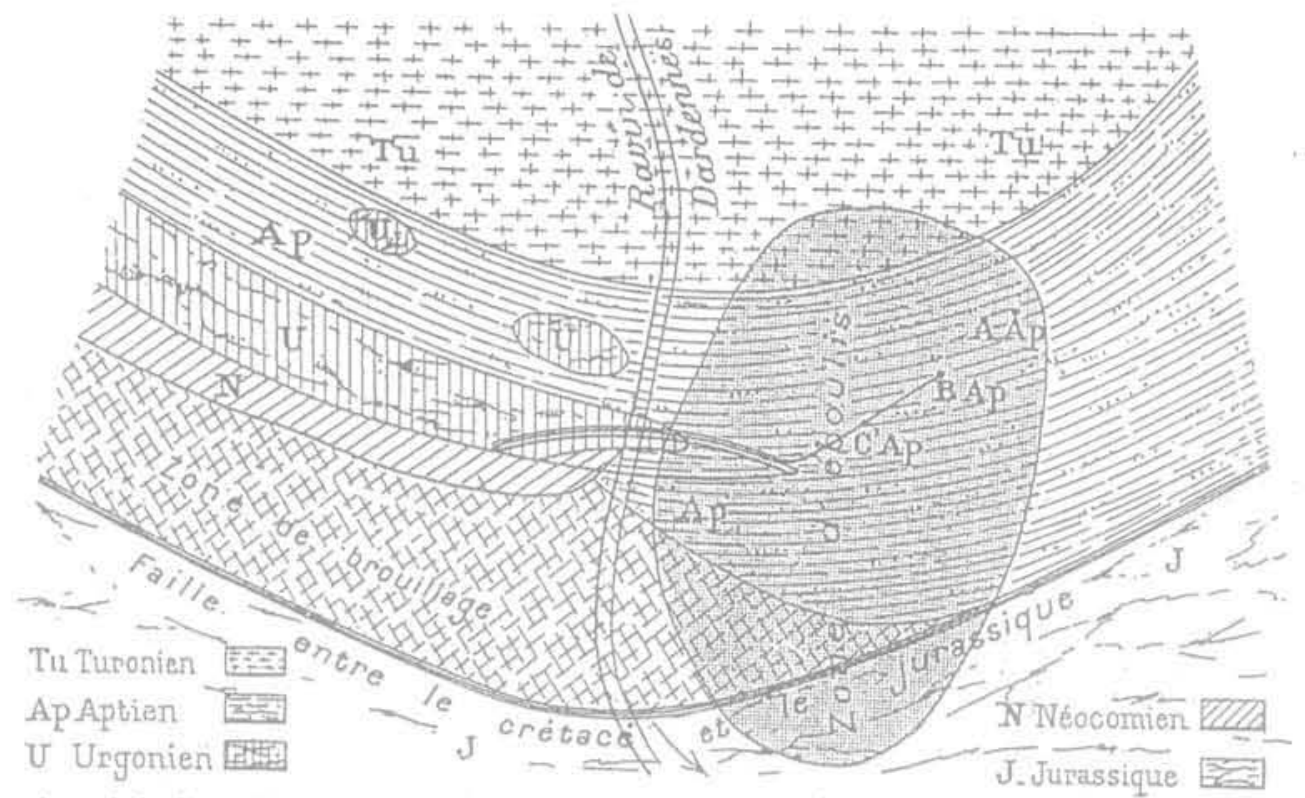

a. Croquis schématique die la disposition des divers terrains aux alentours du barrage et du masque.

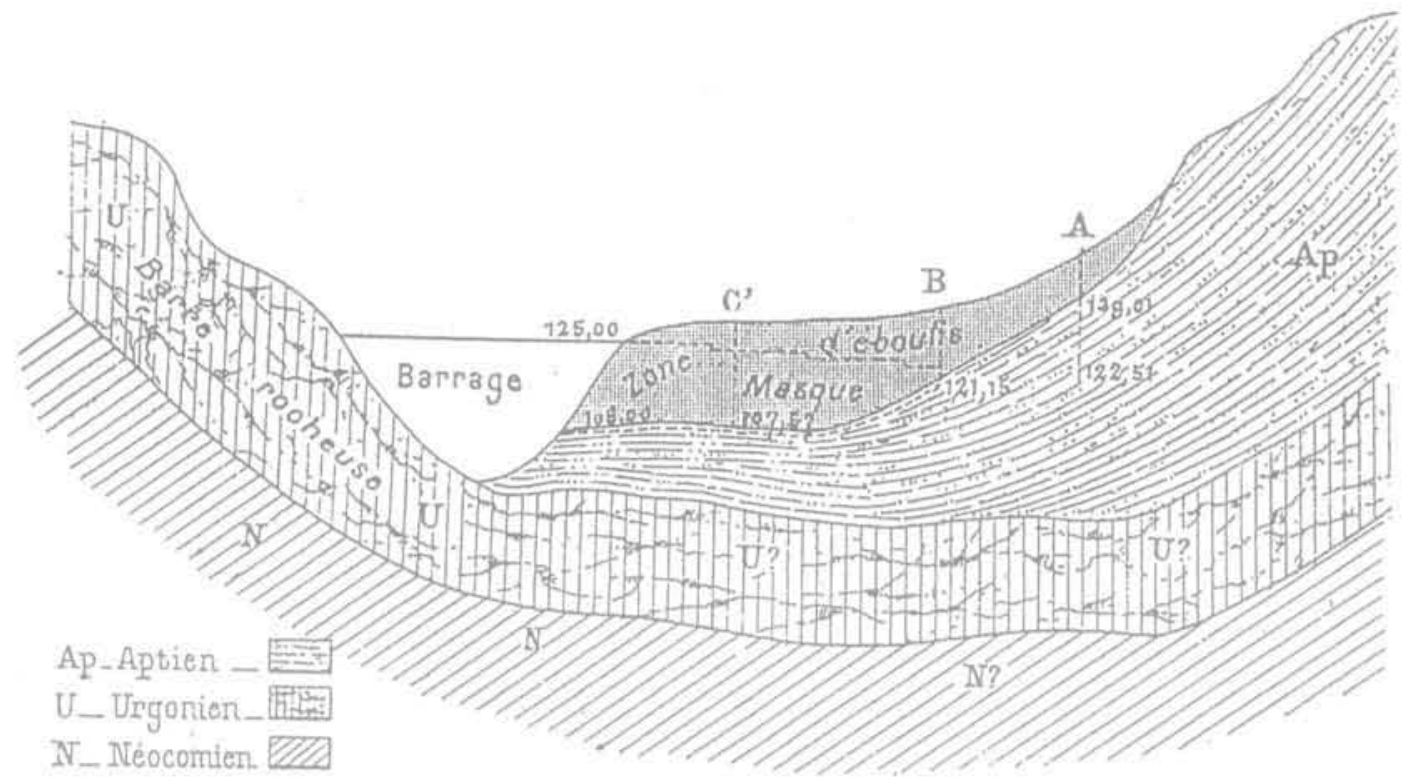

b. Coupe géologique schèmatique suivant le barrage et le masque.

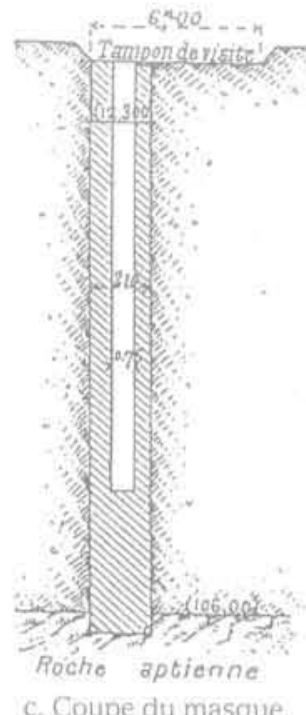

FIG.5 Données géologiques du site du barrage de Dardennes (Le Génie civil, tome LXIV, n" 25, 1914, p. 492).

Geological data of Dardennes dam site. 
TABLEAU II Principales caractéristiques des barrages cités pour la géologie et les injections. Main features of mentionned dams for geology and injection.

\begin{tabular}{|c|c|c|c|c|c|c|c|c|c|c|}
\hline $\begin{array}{l}\text { Nom } \\
\text { du barrage }\end{array}$ & Année & Cours d'eau & $\begin{array}{l}\text { Ville } \\
\text { la pius } \\
\text { proche }\end{array}$ & Département & Type & $\begin{array}{l}\text { Hauteur } \\
\text { (m) }\end{array}$ & $\begin{array}{l}\text { Volume } \\
\text { de la retenue } \\
\left(10^{6} \mathrm{~m}^{3}\right)\end{array}$ & Concepteur & Géologue & Remarques sur les problèmes géologiques \\
\hline Le Lampy & 1782 & Le Lampy & Carcassonne & Aude & Poids & 19 & 1.7 & Garipuy & & $\begin{array}{l}\text { Le site avait èté repéré et mis en balance avec } \\
\text { celui de Saint-Ferréol dès les années } 1670 \text {. }\end{array}$ \\
\hline Grosbois & 1838 & La Brenne & Dijon & Cóte-dror & Poids & 22 & 9,2 & Lacordaire & Lacordaire & $\begin{array}{l}\text { Les ètudes géologiques concernèrent } \\
\text { lensemble de la zone concernée par les cing } \\
\text { barrages d'allmentation du point de partage. } \\
\text { On peut penser que les recherches de carrieres } \\
\text { pour la chaux hydrauliques dans la zone ont } \\
\text { aidé à la connaissance géologique. }\end{array}$ \\
\hline Bouzey & 1881 & L'Avière & Epinal & Vosges & Poids & 22 & 7.1 & Frécot & & $\begin{array}{l}\text { Nous ntavons pas trouvé de nom d'un géo- } \\
\text { logue ou d'un ingénieur ayant acquis une com- } \\
\text { pétence dans ce domaine, qui fut l'auteur des } \\
\text { documents géologiques que nous avons } \\
\text { consultés. }\end{array}$ \\
\hline Dardennes & 1912 & Dardennes & Toulon & Var & Poids & 35 & 1,1 & Boutan & $\begin{array}{l}\text { Bertrand } \\
\text { Kilian } \\
\text { Vasseur } \\
\text { Zurcher }\end{array}$ & \\
\hline Bou Hanifia & 1938 & Hamman & Bou Hanifia & Algérie & $\begin{array}{c}\text { Enrochement } \\
\text { et masque } \\
\text { amont }\end{array}$ & & & Drouhin ot al: & $\begin{array}{l}\text { Falconnier } \\
\text { Lombard } \\
\text { Lugeon } \\
\text { Savornit? }\end{array}$ & $\begin{array}{l}\text { Site gèologique exceptionnellement difficile. } \\
\text { La retenue fut mise en eau à partire de } 1941 \text {. } \\
\text { Noter l'appel qui a été fait à Terzaghi comme } \\
\text { expert pour les problémes de mécanique des } \\
\text { sols. }\end{array}$ \\
\hline Génissiat & 1948 & Le Rhóne & Annecy & $\begin{array}{l}\text { Ain } \\
\text { Haute-Saòne }\end{array}$ & Poids & 104 & 56 & & $\begin{array}{l}\text { Kilian } \\
\text { Lugeon } \\
\text { Martel }\end{array}$ & $\begin{array}{l}\text { Etudes commencées en 1902, mais les études } \\
\text { géologiques, ne commencèrent vraiment que } \\
\text { vers } 1910 \text {. Les noms des géologues cités sont } \\
\text { ceux qui intervinrent avant 1920. }\end{array}$ \\
\hline Serre-Ponçon & 1960 & La Durance & Gap & Ilautes-Alpos & Terre & 129 & 1270 & $\begin{array}{l}\text { Witheim } \\
\text { (jusqu'en 1913) }\end{array}$ & $\begin{array}{l}\text { Kilian } \\
\text { Zurcher }\end{array}$ & $\begin{array}{l}\text { Lets géologues cités sont ceux qui sont interve- } \\
\text { nus jusqu'en } 1917 \text {. L' importance des donnés } \\
\text { géologiques du site à exigé de faire appel aux } \\
\text { meilleur's experts pendant une période très } \\
\text { longue. }\end{array}$ \\
\hline
\end{tabular}


rages avant d'en arriver à une modélisation avec l'introduction des paramètres mécaniques donnés par la mécanique des roches, à partir des années 60 .

Si la connaissance géologique des sites était une des prếoccupations des constructeurs de barrages dès le début de leur activité, avant même que celle-ci ne fût constituée en science, son développement fut laboutisse- ment d'un processus historique. Son application à la construction des barrages ne fit des progrès que sous l'effet d'une demande croissante résultant de l'équipement du pays. Elle fut d'autant plus féconde qu'elle se situa dans le cadre d'une coopération entre les différents acteurs et de transfert technologique des disciplines corinexes et des communautés techniques des pays voisins.

\section{Bibliographie}

Les références qui ont permis d'écrire cet article sont en nombre assez conséquent. Nous n'avons indiqué que les plus importantes ou fes plus significatives

Archives du canal du Midi au service de la navigation Midi-Garonne, borciereau 407, plèce 4, plan de fouille et releve génlogique avec indication de la qualité du rocher, 14 mai 1779

\section{Archives nationales}

Canal de Bourgogne:

Tunnel de Pouilly et données géologiques, Cartes et plans F1410089/3, et cartons Fi46861. Fin6868.

Barrage de Grosbois, carton Fr6861. rap ports de Bonnetat du 12 juillet 1828 et de Lacordaire du $1 *$ mars 1820

Barrage de Bouzey, cartons Fi4 13056 13057, Canal de l'Est. rupture de la đigue de Bouzey, 1897-1898, se référer aussi aux articles du Génie civil des années 1895 et 1897

Barrage de Serre-Ponçon, camons Firy458 à 4460

Aubuisson de Voisins J.F. (d') - Traite de Géognosje: Strasbourg. Levrault, Paris M. le Prince, 1819. 2 vol. $1 \times 1-490$ p. 1 pl. 665 p. 1 pl

Bertrand L. - « Du róle de la géologie dans les études d'aménagements hvdroélec triques x. Volume jubilaire de la Sociéte géotogique de France célébrant le 100 anniversaire 1830-1930, Paris Soclété géologique de France, 1930 1D. $102-118$
Bordes J.L. - a Apercu historique sur la notion de pression de l'eau dans les sols et les milieux fissurès du xyHr au xy siecles en France n. Revue francaise de géotechnique, $n^{\circ} 87$, juin 1999 , p. 3-15.

Bordes J.L. - Regard sur le passé de fa géotechnique x. Revue française de géotechnique, $a^{\circ}$ 91. juin 2000, p. 13-26.

Bordes J.L. - Mobilisation et régulatisation des ressources en eau, les barrages. réservoirs du milieu du Xvin sfècle au début du $x x^{2}$ siécle en France. Thèses pour le doctorat d'histoire de l'université Paris I Panthéon-Sorbonne, sous la direction de Denis Woronoff, soutenue le 22 novembre 2002 , XI-XLV-589. $34 \mathrm{o}$.

Bossut (Abbé) et Viallet - Recherches stir la construction la plus avantageuse des diques. Paris. Ch. A. Jombert. 1764. 1 vol., 60 p., 7 pl:

Boutain, Veilhan, Mercier - " Construction du barrage de Dardennesw. Annaley des Ponts et Chaussées, ge série. tome XIX, janv-fév. 1914. p. 7-74

Delius C.F - Traité sur la science de l'exploitation des mines. Traduit de l'allemand, Philippe-Denys Pierres. Paris. 1778.2 vol.. XXIJ-519 p.. 11 pl et 454 p., 25 plo tome 1. chap 10 « De la construction des étangs in. D. 123-150.

Dumas A. - is L'aménagement hyciraulique du Haut Ahône français, transport electrique de son énercie à Paris is, Le Génie cirll, tome LXI, n" 11, 13 juilles 1912 , p. 214-222.

Dumas A. - « Barrage-réservoir de Dar- dennes pour l'alimentation en eau de la ville de Toulon w., le Génie civil, tome LXIV, $n^{\circ} 25,18$ avril $1914,0,489-493$

Gignoux M. Barbier R. - Géologie des barrages et des aménagements hydraufiques. Paris, Masson et Cie. 1955, 1 vol.. $343 \mathrm{p}$.

Glossop A. - nThe invention and development of injection processes 2. Part 1 . 1802-1850, Getotechnique vol. 10, Sept: 1961, p 91-100: Part II: 1850-1960, Géotechnique vol. 11, Déc. 1962, p. 255-279,

Goguel J. - Application de la géologie aux travaux de l'ingénieur. Masson \& Cie. Paris 1967,1 vol., $376 \mathrm{p}$.

Gohau G. - Une histoire de la géologie. Paris. Le Seuil 1990, 1 vol., 278 p.

Lalande J.f. (de) - Des canaux de navigation et spécialement du canal du Lamquedoc. Vve Desaint, Paris, 1778. réédité en fac similé pour APAMP Toulouse: Grenoble Euromapping, 1996, 1 vol. XIX-586 p.. 14 ol.

Lugeon M. - Barrages et géologie. Réédjtion de l'ouvrage publié en 1933, par lá Société suisse de mécanique des sols et des roches, Montreux 1979, 1 vol. 138 p., 63 photos et 1 carte en hors texte.

Schnitter N. - A history of dams, the useful pyramids. Rotterdam, Balkema, 1994. 1 vol., 266 p.

Wilhelm 1. - La Durance, étucle de l'utilisation cle ses eaux et de l'amélioration de son régime par la création de barrage. Paris, Laveur, Marseille, Jouvène, 1913. 1 vol., 360 p. 